\title{
Gestión de la calidad total y mantenimiento productivo total en la fabricación de alto rendimiento
}

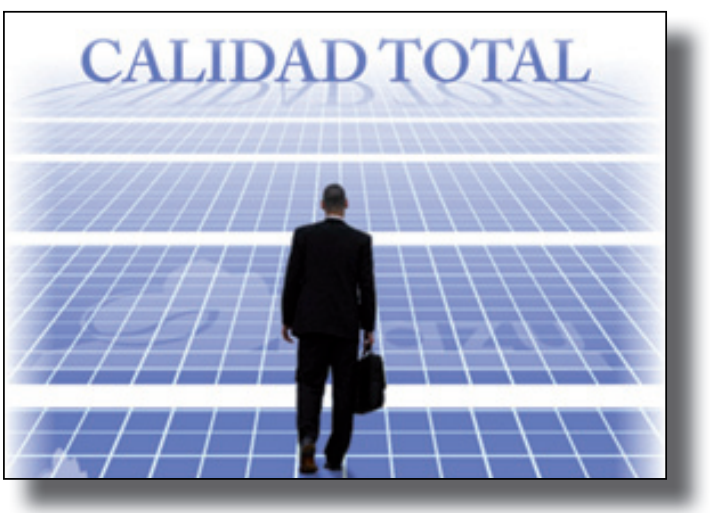

\begin{tabular}{|c|c|}
\hline $\begin{array}{l}\text { Raquel Sanchis } \\
\text { Raul Poler } \\
\text { Josefa Mula } \\
\text { David Peidro }\end{array}$ & $\begin{array}{l}\text { Ingeniera de Organización Industrial } \\
\text { Doctor Ingeniero Industrial } \\
\text { Doctora Ingeniera de Organización Industrial } \\
\text { Doctor Ingeniero de Organización Industrial }\end{array}$ \\
\hline \multicolumn{2}{|c|}{$\begin{array}{l}\text { Universidad Politécnica de Valencia. Centro de Investigación en Gestión e Ingeniería de Producción } \\
\text { (cIGlP). Pl. Ferrándiz y Carbonell, } 2 \text { - } 03801 \text { Alcoy (Alicante). Tfno: }+34963828423 \text {. } \\
\text { rpoler@ @igip.upv.es }\end{array}$} \\
\hline
\end{tabular}

\section{Total quality management and total productive maintenance in high performance manufacturing}

\section{ABSTRACT}

- Introduction

This study compares the implementation's degree of the different methods of the advanced manufacturing practices: Quality Management and Total Productive Maintenance in high performance industrial plants and standard ones to analyze which methods of both practices are correlated with high performance manufacturing.

Material and methods

Based on previous rounds of the international research: "High Performance Manufacturing", a set of questionnaires was developed to perform quantitative analysis of the current situation of industrial plants in Europe, Asia and America in the sectors of machinery, electronics and automotive which conforms the third round of the international research on HPM. The industrial plants' sample distinguished two types: High Performance Manufacturing Plants and Standard Performance Manufacturing Plants. The study used the data provided by 179 plants of the total of 265 participant plants owing to the fact that 86 industrial plants were not classified by the previous types.

Results

The tests carried out provide an overview of which methods related to Quality Management and Total Productive Maintenance present a higher differentiation in the degree of implementation between high performance and standard performance industrial plants. An analysis of the different countries and sectors of the study was performed. Those methods more implemented in industrial plants classified as high performance ones, are those that confer a competitive advantage, although it should be noted that performance is not only a function of the methods analyzed in this study, but also depends on other manufacturing practices of the High Performance Manufacturing model.

Discussion

The methods of process control, customer focus and involvement, and supplier partnership are the techniques most correlated with high performance, as the high performance manufacturing industrial plants have a higher degree of its implementation. Those plants that have also implemented maintenance support show a superior performance than plants that do not consider such an implementation.

- Keywords: Advanced Production Practices, Total Quality Management, Total Productive Maintenance, High Performance Manufacturing, World Class Manufacturing.

\section{RESUMEN}

\section{Introducción}

En este estudio se compara el grado de implementación de diferentes métodos de las prácticas de Gestión de la Calidad y Mantenimiento Productivo Total en diversas plantas industriales, clasificadas como de alto rendimiento o rendimiento estándar, con el objetivo de analizar cuáles son los métodos de ambas prácticas más correlacionados con la fabricación de alto rendimiento.

Material y métodos

A partir de dos rondas anteriores del proyecto internacional High Performance Manufacturing (HPM), se desarrollaron un conjunto de cuestionarios que sirvieron para realizar el análisis cuantitativo de la situación actual de las plantas industriales de Europa, Asia y Estados Unidos de los sectores de maquinaria, electrónica y automoción en lo que acabó siendo la tercera ronda internacional HPM. La muestra de plantas industriales distinguía dos tipologías: Plantas de Alto Rendimiento (PAR) y Plantas de 
Rendimiento Estándar (PRE). El estudio utilizó la información proporcionada por 179 plantas del total de 265 plantas participantes debido a que 86 plantas industriales no fueron clasificadas según su tipología en PAR y PRE.

\section{Resultados}

Las comparativas desarrolladas ofrecen una visión de cuáles son los métodos de Gestión de la Calidad y Mantenimiento Productivo Total en los que existe una mayor diferenciación en el grado de implementación entre plantas industriales de alto rendimiento y de rendimiento estándar. También, se realiza un análisis de los diferentes países del estudio y sectores. Aquellos métodos más implantados en las plantas industriales clasificadas como de alto rendimiento son los que confieren una ventaja competitiva, aunque cabe destacar que el rendimiento no sólo es función de los métodos analizados en el presente estudio, sino que también depende de otras prácticas del modelo de Fabricación de Alto Rendimiento.

\section{Discusión}

Los métodos de control de procesos, orientación al cliente y asociación con proveedores son las técnicas más correlacionadas con el alto rendimiento, ya que las plantas industriales de alto rendimiento tienen un mayor grado de implementación de los mismos. Aquellas plantas que también poseen mantenimiento de apoyo implantado presentan un mayor rendimiento que las plantas que no lo consideran.

Palabras clave: Prácticas Avanzadas de Producción, Gestión de la Calidad Total, Mantenimiento Productivo Total, Fabricación de Alto Rendimiento, Fabricación de Clase Mundial.

\section{INTRODUCCIÓN}

El incremento de la competencia mundial en plantas industriales desvela la necesidad de desarrollar capacidades de producción que permitan a las organizaciones una ventaja competitiva sostenible sobre sus competidores. Se precisa de la habilidad para adaptarse a las necesidades de los clientes y a los requerimientos cambiantes del entorno. Por ello, una mayor focalización en la gestión de los procesos de fabricación pretende contribuir en el incremento del rendimiento de la fabricación. Schroeder y Flynn (2001) definen la Fabricación de Alto Rendimiento (HPM, High Performance Manufacturing) también conocida como Fabricación de Clase Mundial (WCM, World Class Manufacturing), como un conjunto integrado de procesos diseñados para alcanzar una ventaja competitiva mundial sostenible por medio de la mejora continua de la capacidad de fabricación. La mejora continua implica la utilización de prácticas avanzadas de producción. El modelo actual de HPM abarca diferentes prácticas avanzadas como: Justo a Tiempo (JIT, Just In Time), Gestión de la Calidad Total (TQM, Total Quality Management), Gestión de los Recursos Humanos, Mantenimiento Productivo Total (TPM, Total Productive Maintenance), Sistemas de Información, Estrategia de
Fabricación, Desarrollo de Nuevos Productos, Gestión de la Cadena de Suministro y Gestión de la Tecnología (Schroeder y Flynn, 2001; Forza y Salvador, 2001).

El presente artículo se centra en el estudio de la relación existente entre laHPMy los métodos de TQMyTPMen plantas industriales de Europa, Asia y Estados Unidos. La naturaleza industrial de las plantas incluye tanto a Plantas de Alto Rendimiento (PAR) como Plantas de Rendimiento Estándar (PRE). Las PAR son plantas que tienen implementadas y demostradas las mejores prácticas avanzadas del sector. Son aquellas plantas líderes y referentes en sus procesos productivos, que poseen una ventaja competitiva probada así como la capacidad de absorber cambios de la mejor manera para incrementar sus posibilidades de permanecer en los mercados (local, nacional e internacional).

TQM es un sistema integrado de fabricación dirigido a mejorar continuamente y mantener productos y procesos de calidad mediante la gestión empresarial, los recursos humanos, proveedores y clientes con el fin de alcanzar e incluso superar las expectativas y necesidades de los clientes (Hackman y Wageman, 1995; Powell, 1995; Cua, McKnoe y Schroeder, 2001).

El modelo HPM, que está relacionado con los principios de la Producción Ajustada, engloba la práctica avanzada de TQM, que se centra en los siguientes métodos de gestión de la calidad total (Ishikawa, 1985; Juran y Gryna, 1988): (i) participación de proveedores en el proceso de calidad, (ii) participación de clientes, (iii) control de procesos, (iv) énfasis en los procesos, (v) orientación al cliente, (vi) TQM conectada a clientes, (vii) satisfacción de los clientes, (viii) enfoque amplio organizativo, (ix) prevención, (x) planificación estratégica de la calidad, (xi) mejora continua y aprendizaje, (xii) limpieza y organización y (xiii) retroalimentación.

TPM se define como un programa de fabricación diseñado principalmente para maximizar la efectividad de los equipos de fabricación en toda su vida útil a través de la participación y motivación de todos los recursos humanos de la empresa (Nakajima, 1988). Espolita-Carreño (2000) afirmaba que "donde quiera que existan activos físicos, se generan actividades de restauración consecuencia de las averías, y ello ocasiona costes de reparar, indisponibilidad de los equipos y riesgos de daños a personas, a las cosas y al medio ambiente". Por ello, se precisa de un programa de fabricación diseñado principalmente para garantizar y controlar la disponibilidad de los equipos productivos y la calidad del producto terminado a un costo mínimo (Galar-Pascual et al., 2010). Los métodos de TPM, también considerados como característicos de la Producción Ajustada, que han sido analizados en el presente estudio son los que se detallan a continuación (Ohno, 1988 y Denis, 2002): (i) mantenimiento autónomo, (ii) mantenimiento preventivo, (iii) mantenimiento de apoyo y (iv) equipos de mantenimiento.

La HPM usa TQM y TPM como medios para controlar y mejorar el proceso de producción con el fin de satisfacer 
las necesidades de los clientes y así ganar una ventaja competitiva (Flynn, Schroeder y Flynn, 1999), además de eliminar defectos y reducir la reelaboración de productos, ayudando a mejorar la calidad y la entrega de dichos productos y servicios (Cua, McKnoe y Schroeder, 2001). Rodriguez-Darnes (2000) afirma que la calidad de los procesos está unida al buen funcionamiento de la maquinaria con lo cual la gestión de calidad de los procesos junto al mantenimiento productivo hace que los equipos funcionen eficaz y eficientemente obteniendo productos de calidad alta.

El presente estudio internacional tiene como objetivo, por una parte, diseminar a la industria en general las conclusiones obtenidas en el estudio que servirán:

- Como autoevaluación del estado AS IS de las plantas industriales participantes para así poder analizar su estado respecto a las plantas de su mismo sector y país, y poder realizar comparativas de su rendimiento con otras plantas industriales de otros sectores y otros países. Se trata de mostrar a las plantas cuáles son los métodos de TPM y TQM que mayoritariamente están implantados en las PAR y que no lo están en las PRE. Dichas diferencias pueden dar una orientación de qué métodos están más correlacionados con el alto rendimiento y por ello, su implantación puede llevar a las plantas a obtener una ventaja competitiva respecto a aquellas plantas que no tienen dichos métodos establecidos. Al final del estudio, las plantas participantes recibieron un informe comparativo.

- Basándose en el análisis anterior, las plantas industriales podían extraer conclusiones interesantes acerca de su estado y de cuáles podrían ser las directrices que debían seguir para maximizar su rendimiento y de esta forma definir su estado $T O B E$.

Por otra parte, se pretende que los resultados del estudio HPM se difundan en el ámbito académico para contribuir al desarrollo colectivo de la investigación a nivel internacional.

\section{MATERIAL Y MÉTODOS}

El estudio está enmarcado dentro de la tercera ronda del proyecto internacional sobre HPM para la creación de una base de datos intercontinental. La primera ronda se realizó en los años 80 y tan sólo abarcó a plantas industriales de los sectores de maquinaria, electrónica y automoción de diferentes regiones de los Estados Unidos. En dicha ronda no había una definición de los perfiles de los encuestados, aunque comprendió desde mano de obra directa hasta la gerencia de las plantas industriales. Las cuestiones planteadas englobaban tanto aspectos cualitativos como cuantitativos sobre el entorno, principales productos, organización, recursos humanos, gestión de la calidad total, sistemas de información, JIT, estrategias de fabricación, desarrollo de tecnologías, y mejora y rendimiento.

La segunda ronda del proyecto internacional HPM se realizó durante los años 90 y en ella se incrementó el número de países participantes, ya que plantas industriales de Japón, Estados Unidos, Reino Unido, Alemania e Italia fueron encuestadas. Los sectores analizados fueron los mismos que en la primera ronda y que en el presente estudio: maquinaria, electrónica y automoción. Se definió de forma concisa los perfiles de los encuestados que fueron 26 y se analizaron 2544 aspectos cualitativos y cuantitativos sobre la temática anterior.

\begin{tabular}{|c|c|c|c|}
\hline & $\begin{array}{l}\text { Primera } \\
\text { Ronda }\end{array}$ & $\begin{array}{l}\text { Segunda } \\
\text { Ronda }\end{array}$ & Tercera Ronda (actual) \\
\hline Año & $80 \mathrm{~s}$ & $90 \mathrm{~s}$ & $2000 \mathrm{~s}$ \\
\hline Países & $\begin{array}{l}\text { Estados } \\
\text { Unidos }\end{array}$ & $\begin{array}{l}\text { Japón, } \\
\text { Estados } \\
\text { Unidos, } \\
\text { Reino Unido, } \\
\text { Alemania e } \\
\text { Italia }\end{array}$ & $\begin{array}{l}\text { Alemania, Austria, Corea } \\
\text { del Sur, España, Estados } \\
\text { Unidos, Finlandia, Italia, } \\
\text { Japón y Suecia }\end{array}$ \\
\hline Sector & \multicolumn{3}{|c|}{ Automoción, Maquinaria y Electrónica } \\
\hline $\begin{array}{l}\text { Número de } \\
\text { plantas }\end{array}$ & 45 & 164 & 265 \\
\hline Encuestados & Varios & 26 & 21 \\
\hline Cuestiones & \multicolumn{3}{|c|}{ Aspectos cualitativos y cuantitativos } \\
\hline $\begin{array}{l}\text { Temática } \\
\text { analizada }\end{array}$ & \multicolumn{2}{|c|}{$\begin{array}{l}\text { Entorno, principales } \\
\text { productos, organización, } \\
\text { recursos humanos, gestión } \\
\text { de la calidad total, sistemas } \\
\text { de información, JIT, } \\
\text { estrategias de fabricación, } \\
\text { desarrollo de tecnologías, y } \\
\text { mejora y rendimiento. }\end{array}$} & $\begin{array}{l}\text { Justo a Tiempo (JIT) y } \\
\text { Teoría de Restricciones } \\
\text { (TOC), Gestión de la } \\
\text { Calidad Total (TOM), } \\
\text { Gestión de los Recursos } \\
\text { Humanos (RRHH), } \\
\text { Mantenimiento Productivo } \\
\text { Total (TPM), Sistemas } \\
\text { de información (TIC), } \\
\text { Estrategia de Fabricación, } \\
\text { Desarrollo de Nuevos } \\
\text { Productos (NPD), } \\
\text { Gestión de la Cadena } \\
\text { de Suministro (SCM) y } \\
\text { Tecnología (Tec.). }\end{array}$ \\
\hline
\end{tabular}

Tabla 1: Resumen de las rondas del estudio internacional HPM

Los datos utilizados se recogieron como parte de la tercera ronda del proyecto: "High Performance Manufacturing (HPM)” (Fabricación de Alto Rendimiento), que se basó en las experiencias obtenidas en las dos rondas anteriores, así como en una revisión extensa de la literatura relevante sobre HPM y fue llevado a cabo por un equipo de investigadores de diversas universidades de los Estados Unidos, Europa y Asia. El equipo español del estudio estaba formado por grupos de investigación de cuatro universidades: Universidad de Sevilla, Universidad Politécnica de Valencia, Universidad Complutense de Madrid y Universidad Pública de Navarra, abarcando todas las zonas geográficas del país. La metodología utilizada es correlacional dentro del estudio no experimental sobre HPM, ya que trata de visualizar cómo se relacionan o vinculan diversos fenómenos entre sí, es decir se evalúa el grado de relación entre dos variables y en este caso el estudio analiza el grado de implementación de los métodos TQM y TPM 
y la relación que dichos métodos tienen en el rendimiento de las plantas industriales (distinguiendo dos tipologías de plantas industriales: PAR y PRE). La información fue recogida mediante cuestionarios en 265 plantas industriales. Para analizar la fiabilidad, validez y consistencia interna, se realizaron diferentes pruebas estadísticas y no estadísticas como el alfa de Cronbach (1951), la medida de fiabilidad conceptual de Fornell y Larcker (1981), el análisis del factor confirmatorio y correlaciones, pares entre otras.

La muestra de plantas industriales distinguía dos tipologías: Plantas de Alto Rendimiento (PAR) y Plantas de Rendimiento Estándar (PRE). En España no existe ninguna base de datos que recoja las principales PAR en el área de producción/operaciones. Por ello, y tras un profundo análisis, se acordó usar como fuente de identificación de las PAR, el directorio Duns, que considera estas plantas como las principales en España por volumen de facturación. Se escogió la muestra de forma aleatoria simple del 35\% de las primeras plantas del listado Duns. Además, cabe destacar que dichas plantas también aparecen catalogadas como líderes por otras fuentes (Revista de Manutención y Almacenaje, Price Waterhouse Coopers, Industry Week). La identificación de las PRE incluyó el 65\% restante del listado de Duns. Además se utilizaron otras fuentes de información como: AETIC, AFM, DBK, Europages, Fábricas de España, Guíame, Kompas, SERNAUTO, SABI y Thomas Global Register, para la determinación de las PRE. A nivel internacional, la distinción de ambas tipologías de plantas se realizó clasificando las PAR como aquellas plantas que habían recibido un reconocimiento público en forma de premios internacionales utilizando listados de prestigiosas revistas como Shingo Prize Award, Deming Prize e Industry Week Best Plants Award. El presente estudio recogió la información de 265 plantas participantes de Alemania, Austria, Corea del Sur, España, Estados Unidos, Finlandia, Italia, Japón y Suecia, aunque tan sólo se utilizó la información proporcionada por 179 plantas del total debido a que las plantas industriales alemanas, austriacas y suecas no fueron clasificadas según su tipología en PAR y PRE.

A las plantas que manifestaron su interés en participar en el estudio, se les distribuía un conjunto de 21 cuestionarios para que fueran cumplimentados por empleados de diferente nivel organizativo y con diversas funciones, siendo éstos: 10 directores de diferentes áreas (planta, producción, contabilidad, recursos humanos, control de producción, calidad, sistemas de información, materiales (inventario), procesos y desarrollo de productos), 6 supervisores de planta y 5 operarios.

Finalmente, se procedió a la codificación (para asegurar la confidencialidad) y entrada de la información en la base de datos HPM y al análisis de los resultados.

Detalles adicionales de los procedimientos de recopilación de datos se puede encontrar en Huang et al. (2008); y Sanchis et al. (2009 y 2010). Cerca del $60 \%$ de las plantas industriales españolas contactadas decidieron participar en el estudio y suministraron la información pertinente mediante la cumplimentación de los cuestionarios para su análisis (Ortega, 2007).

\section{RESULTADOS}

El modelo actual de HPM engloba diferentes tipos de prácticas avanzadas. En el presente estudio se analiza el grado de implementación de las prácticas avanzadas de producción de TQM y TPM tanto en PAR como en PRE, con el fin de cuantificar el rendimiento global de las diferentes plantas y comparar ambos tipos, quedando fuera del ámbito de este artículo el estudio y análisis del grado de utilización de otras prácticas avanzadas del modelo HPM. Se puede encontrar información adicional sobre estudios del proyecto HPM que analizan las diferentes prácticas en Bozarth et al. (2009), que analizan la gestión de la cadena de suministro; Hallgren y Olhager (2009), que exploran las estrategias de fabricación y Ortega et al. (2009), que estudian las prácticas de estrategias de fabricación y tecnología. La Tabla 2 recoge diferentes promedios de una serie de medibles económicos y de capacidad que evidencian las diferencias entre las PAR y las PRE.

\begin{tabular}{|c|c|c|c|}
\hline Medible & PAR & PRE & $\begin{array}{l}\% \text { PAR } \\
\text { vs PRE }\end{array}$ \\
\hline Valor de las ventas por producción anuales (€ 000) & 532.391 & 283.140 & $+88 \%$ \\
\hline Costes de fabricación anuales $(€ 000)$ & 307.289 & 232.626 & $+32 \%$ \\
\hline $\begin{array}{l}\text { Porcentaje de los costes de fabricación = mano de } \\
\text { obra directa }(\%)\end{array}$ & $11,96 \%$ & $17,42 \%$ & $-31 \%$ \\
\hline $\begin{array}{l}\text { Porcentaje de los costes de fabricación }= \\
\text { Materiales }(\%)\end{array}$ & $70,58 \%$ & $63,67 \%$ & $+11 \%$ \\
\hline $\begin{array}{l}\text { Porcentaje de los costes de fabricación = Gastos } \\
\text { generales }(\%)\end{array}$ & $17,46 \%$ & $18,90 \%$ & $-8 \%$ \\
\hline $\begin{array}{l}\text { Porcentaje de los materiales procedentes de fuera } \\
\text { de la empresa }\end{array}$ & $77,00 \%$ & $86,00 \%$ & $-10 \%$ \\
\hline $\begin{array}{l}\text { Porcentaje de los materiales procedentes de fuera } \\
\text { del pais }\end{array}$ & $28,50 \%$ & $25,70 \%$ & $+11 \%$ \\
\hline $\begin{array}{l}\text { Valor de los inventarios (promedio anual) }= \\
\text { Productos terminados }(€ 000)\end{array}$ & 9.064 & 4.783 & $+89 \%$ \\
\hline $\begin{array}{l}\text { Valor de los inventarios (promedio anual) = trabajo } \\
\text { en proceso }(€ 000)\end{array}$ & 9.073 & 4.844 & $+87 \%$ \\
\hline $\begin{array}{l}\text { Valor de los inventarios (promedio anual) = } \\
\text { Materias primas }(€ 000)\end{array}$ & 25.851 & 6.758 & $+283 \%$ \\
\hline Inversión neta en planta y equipo (€ 000) & 56.730 & 44.179 & $+28 \%$ \\
\hline Inversión anual en instalaciones y equipo (€ 000) & 19.357 & 7.673 & $+152 \%$ \\
\hline Número anual de horas de operación de la planta & 4.167 & 3.495 & $+19 \%$ \\
\hline Número de personal temporal empleado & 507 & 281 & $+80 \%$ \\
\hline Número de personal fijo empleado & 469 & 316 & $+48 \%$ \\
\hline Tiempo de ciclo total (días) & 30 & 33 & $-9 \%$ \\
\hline Tiempo medio de entrega (días) & 39 & 30 & $+30 \%$ \\
\hline Porcentaje de órdenes entregadas a tiempo & $91 \%$ & $88 \%$ & $+3 \%$ \\
\hline Espacio utilizado por la planta (metros cuadrados) & 634.780 & 82.598 & $+669 \%$ \\
\hline
\end{tabular}

Tabla 2: Comparativa de promedios de medibles económicos y de capacidad entre PAR y PRE

Como se deriva de la comparativa anterior, si bien las PAR arrojan un tamaño medio mayor, la muestra de PRE se compone de empresas de un tamaño suficiente para minimizar 
la influencia de dicha característica. Además, los costes de fabricación de mano de obra directa son en general menores en las PAR, lo que podría deberse a la implementación en sus plantas de prácticas avanzadas de producción, y a que su porcentaje de pedidos entregados a tiempo es ligeramente superior respecto a las PRE.

Los cuestionarios de cada planta fueron agregados para obtener las valoraciones definitivas sobre cada uno de los métodos de las prácticas de TQM y TPM. Se realizaron diferentes análisis estadísticos con la finalidad de averiguar la relación existente entre la implementación de determinados métodos de TQM y TPM y el rendimiento de las plantas. Se definieron 4 niveles para la agrupación de la escala relativa al grado de implementación de los métodos de cada práctica según la media de los valores $+/-1$ de desviación típica definiendo 3 puntos de corte. El nivel 1, es aquel en el que las empresas poseen un grado bajo de implementación, en el nivel 2, el grado de implementación es medio, y los niveles 3 y 4 muestran los grados de implementación altos y muy altos, respectivamente. Centrándose en el nivel 4, en el cual el grado de implementación de los métodos de TQM y TPM es el más alto, se calcularon las diferencias porcentuales entre PAR y PRE, lo cual ofrece una visión de en qué métodos existe una mayor diferencia entre su grado de implementación entre ambos tipos de plantas. Las Figs. 1 y 2 muestran dichas diferencias.

Se puede observar como las PAR poseen un grado de implementación de los métodos TQM superior a las PRE. Los métodos de TQM de control de procesos, orientación al cliente, asociación con proveedores y TQM conectada con los clientes, son las técnicas más correlacionadas con el alto

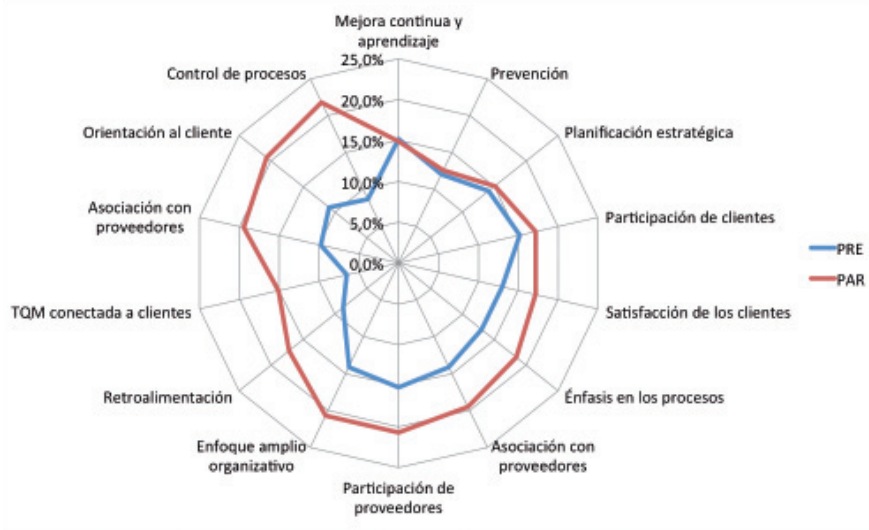

Figura 1: Resultados comparativos entre PAR y PRE de métodos de TQM

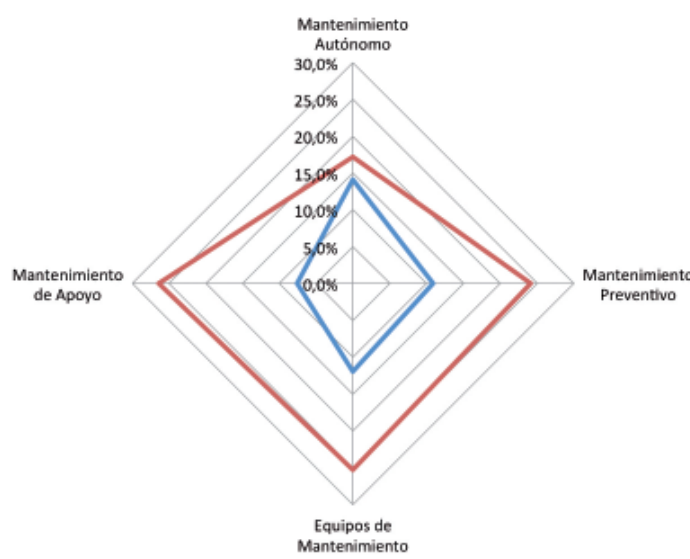

Figura 2: Resultados comparativos entre PAR y PRE de métodos de TPM

\begin{tabular}{|c|c|c|c|c|c|c|c|}
\hline & Corea del Sur & España & EEUU & Finlandia & Italia & Japón \\
\hline \multirow{14}{*}{ TQM } & Limpieza y organización & & & B & B & & \\
\hline & Mejora continua y aprendizaje & & & C & & & \\
\hline & Orientación al cliente & & & & A & & \\
\hline & Participación de clientes & & A & & & & \\
\hline & Satisfacción de los clientes & C & & & & C & \\
\hline & Retroalimentación & B & & & & & A \\
\hline & Enfoque amplio organizativo & & & & & & $\mathrm{B}$ \\
\hline & Prevención & & & & & & \\
\hline & Control de procesos & A & & & & & C \\
\hline & Énfasis en los procesos & & & A & & & \\
\hline & Asociación con proveedores & & & & A & B & \\
\hline & $\begin{array}{l}\text { Participación de proveedores en el } \\
\text { proceso de calidad }\end{array}$ & & & & B & C & \\
\hline & Planificación estratégica de la calidad & & B & & & A & \\
\hline & TQM conectada a clientes & & c & & & & \\
\hline \multirow{4}{*}{ TPM } & Mantenimiento Autónomo & B & C & & C & & C \\
\hline & Mantenimiento Preventivo & A & B & C & B & C & B \\
\hline & Mantenimiento de apoyo & & A & A & A & A & A \\
\hline & Equipos de Mantenimiento & C & & B & & B & \\
\hline
\end{tabular}

Tabla 3: Ranking de los métodos de TQM y TPM según los diferentes países del estudio 
rendimiento, mientras que no existen diferencias significativas en los métodos de mejora continua y aprendizaje, prevención y planificación estratégica de la calidad. Por tanto, cabe destacar que estos tres últimos métodos son utilizados con el mismo grado de implementación entre las diferentes plantas.

En el caso de la práctica avanzada de TPM, el grado de implementación del método de mantenimiento de apoyo es el que en mayor medida se diferencia entre las PAR y PRE, aunque también cabe destacar que existen diferencias significativas en los métodos de mantenimiento preventivo y equipos de mantenimiento. El grado de implementación del mantenimiento autónomo es muy similar tanto en PAR como en PRE.

Con el objetivo de identificar diferencias entre las empresas en los diferentes países del estudio, se realizó una comparativa entre las PAR y PRE de los métodos de TQM y TPM cotejando la información de los métodos implementados. Tal y como se ha comentado anteriormente, los datos de los cuestionarios fueron clasificados en 4 niveles. El nivel 1, es aquel en el que las empresas poseen un grado bajo de implementación, en el nivel 2, el grado de implementación es medio, y los niveles 3 y 4 muestran los grados de implementación altos y muy altos respectivamente de métodos TQM y TPM. En la comparativa por países, el objetivo es contrastar las diferencias existentes entre PAR y PRE en el nivel 4 (mayor grado de implantación). En la Tabla 3 se muestra el ranking de los métodos de TQM y TPM en los que existen mayores diferencias en su nivel de implementación entre PAR y PRE, según los países participantes. El valor A significa que es el método que mayores diferencias presenta en el grado de implementación en el nivel 4 entre PAR y PRE, seguido de los valores B y C.

Como se puede observar, el método de TPM más ampliamente utilizado por las PAR de casi todos los países participantes es el de mantenimiento de apoyo, mientras que en el caso de los métodos de TQM, existe una gran dispersión en el grado de implementación de los diferentes métodos según los países del estudio.

Respecto a la dimensión sectorial (Tabla 4), se observa que la ventaja competitiva de las PAR en los sectores de la electrónica y maquinaria se debe principalmente al método de TQM de retroalimentación, mientras que en el caso del sector de automoción, el alto rendimiento de las plantas de Clase Mundial se debe a su orientación al cliente. Y, en relación con los métodos de TPM, casi todos los sectores presentan que el mantenimiento de apoyo es el método que ofrece una mayor correlación con la HPM.

Con objeto de comprobar para qué métodos de TQM y TPM las dos muestras de PAR y PRE presentan medias diferenciadas, se aplicó la prueba de Levene para la igualdad de varianzas y la prueba $\mathrm{T}$ para la igualdad de medias obteniendo que los métodos para los que se aprecia una diferencia en las medias coinciden con el análisis comparativo anterior.

Se realizaron también las mismas pruebas estadísticas para los diferentes medibles de rendimiento de las plantas industriales, estudiando las diferencias de rendimiento entre PAR y PRE, obteniendo que las medidas de rendimiento en negrita y subrayadas de la Tabla 5 son las que presentan mayores diferencias.

Las medidas de rendimiento que mayores diferencias presentan entre PAR y PRE son la innovación de los productos y el apoyo al cliente. Ambas medidas están muy relacionadas con los métodos de TQM y TPM, que también presentan una gran diferenciación entre PAR y PRE. Desde la perspectiva de calidad, dichos métodos son de orientación al cliente y TQM conectada con clientes, cuyo objetivo es dirigirse hacia el cliente para conocer sus necesidades y de esta forma desarrollar productos innovadores que cumplan sus requisitos. Desde la perspectiva del mantenimiento, los métodos que mayores diferencias en el grado de implementación presentan entre PAR y PRE son: mantenimiento de apoyo $y$ equipos de mantenimiento. Estos últimos se centran en equipos multi-funcionales expertos en estudiar la viabilidad de los equipos para la fabricación de nuevos productos innovadores que satisfagan las necesidades de los clientes y que, por tanto, contribuyan a las medidas

Tabla 4: Ranking de los métodos de TQM y TPM según los diferentes sectores del estudio: Au (Automoción), E (Electrónica) y M (Maquinaria). 


\begin{tabular}{|c|c|c|c|c|c|c|c|}
\hline \multirow{3}{*}{ Medidas de rendimiento } & \multicolumn{7}{|c|}{ Prueba T para la igualdad de medias } \\
\hline & \multirow[t]{2}{*}{$\mathrm{t}$} & \multirow[t]{2}{*}{ gl } & \multirow{2}{*}{$\begin{array}{l}\text { Sig. } \\
\text { (bilateral) }\end{array}$} & \multirow{2}{*}{$\begin{array}{l}\text { Diferencia } \\
\text { de medias }\end{array}$} & \multirow{2}{*}{$\begin{array}{l}\text { Error típ. de } \\
\text { la diferencia }\end{array}$} & \multicolumn{2}{|c|}{$\begin{array}{l}\text { 95\% Intervalo de confianza } \\
\text { para la diferencia }\end{array}$} \\
\hline & & & & & & Inferior & Superior \\
\hline Fabricación por unidad de coste & 1,76 & 153 & 0,08 & 0,23202 & 0,13160 & $-0,02798$ & 0,49201 \\
\hline $\begin{array}{l}\text { Conformidad con las especificaciones del } \\
\text { producto }\end{array}$ & 2,55 & 161 & $\underline{0,012}$ & 0,28345 & 0,11131 & 0,06364 & 0,50327 \\
\hline Rendimiento en el tiempo de entrega & 2,57 & 161 & $\underline{0,011}$ & 0,33554 & 0,13037 & 0,07810 & 0,59299 \\
\hline Entrega rápida & 0,63 & 162 & 0,532 & 0,08333 & 0,13314 & $-0,17958$ & 0,34625 \\
\hline $\begin{array}{l}\text { Flexibilidad en los cambios en el mix de } \\
\text { productos }\end{array}$ & 2,46 & 159 & $\underline{0,015}$ & 0,28684 & 0,11672 & 0,05632 & 0,51736 \\
\hline Rotación de inventarios & 2,31 & 156 & $\underline{0,022}$ & 0,31346 & 0,13563 & 0,04555 & 0,58137 \\
\hline $\begin{array}{l}\text { Ciclo de vida (desde las materias primas hasta la } \\
\text { entrega de productos) }\end{array}$ & 1,71 & 156 & 0,089 & 0,20032 & 0,11723 & $-0,03125$ & 0,43189 \\
\hline $\begin{array}{l}\text { Velocidad en la introducción de nuevos productos } \\
\text { en la planta }\end{array}$ & 1,93 & 158 & 0,056 & 0,27500 & 0,14258 & $-0,00660$ & 0,55660 \\
\hline Capacidad y rendimiento de los productos & 2,15 & 159 & $\underline{0,033}$ & 0,25864 & 0,12060 & 0,02046 & 0,49682 \\
\hline Lanzamiento a tiempo de nuevos productos & 2,29 & 156 & $\underline{0,024}$ & 0,31987 & 0,13995 & 0,04343 & 0,59631 \\
\hline Innovación de los productos & 2,98 & 157 & $\underline{0,003}$ & 0,43354 & 0,14549 & 0,14618 & 0,72091 \\
\hline Apoyo y servicio al cliente & 2,64 & 162 & $\underline{0,009}$ & 0,35476 & 0,13447 & 0,08921 & 0,62031 \\
\hline
\end{tabular}

Tabla 5: Prueba T para la igualdad de medias de las diferentes medidas de rendimiento

de rendimiento anteriores. La conformidad con las especificaciones del producto y el lanzamiento a tiempo de nuevos productos son otras medidas de rendimiento que están alineadas con los métodos de TQM y TPM mencionados arriba y que mayores diferencias presentan en cuanto a la tipología de plantas industriales.

Las medidas de rendimiento según el país y sector siguen la misma tendencia observada en el análisis anterior y están alineadas a las diferencias entre el grado de implementación de los métodos de TQM y TPM entre PAR y PRE.

\section{DISCUSIÓN}

El análisis realizado muestra que existen diferencias significativas entre el grado de implementación de métodos TQM y TPM entre la diferente tipología de plantas industriales. Dichas diferencias varían según el sector analizado siendo más relevantes en el sector de la automoción y la electrónica y muy escasas en el sector de la maquinaria, lo cual da a entender que las plantas industriales de los dos primeros sectores poseen una implementación mayor de ciertos métodos de TQM y TPM y esto impacta directamente en su mayor rendimiento de fabricación frente a aquellas que no poseen ciertos métodos implantados o los tiene en menor medida y por ello su rendimiento en inferior. Dichas diferencias también varían según el país estudiado, siendo Corea del Sur y Estados Unidos los que presentan un mayor porcentaje de diferenciación en términos absolutos en el grado de implementación de los métodos de TQM, entrando Italia también en el ranking de diferenciación para los métodos de TPM.

Por todo ello, cabe destacar que las plantas industriales correlacionadas con el alto rendimiento suelen tener altos niveles de implementación de los métodos de control de procesos, orientación al cliente, asociación con proveedores y TQM conectada con clientes (TQM) así como el mantenimiento de apoyo (TPM), hecho que no presentan las plantas industriales estándares.

Por último, señalar que también se encuentran diferencias significativas en las variables de rendimiento por sectores, encontrando mayores diferencias en el sector de la automoción, seguido del sector de la electrónica y siendo casi inexistentes en el sector de maquinaria. Las diferencias en los medibles de rendimiento siguen la misma tendencia que el grado de implementación de métodos TQM y TPM, aunque cabe destacar que en el presente estudio tan sólo se ha estudiado el impacto de dos prácticas avanzadas de producción y los medibles de rendimiento también son función del grado de implementación de otras prácticas avanzadas de producción del modelo actual de HPM como serían por ejemplo Justo a Tiempo, Gestión de los Recursos Humanos, Sistemas de Información, Estrategia de Fabricación, Desarrollo de Nuevos Productos, Gestión de la Cadena de Suministro y Gestión de la Tecnología. Por ello, un análisis combinado de la implantación de todas las prácticas ofrecería una mejor visión de su impacto en el rendimiento de las plantas y de las diferencias observadas entre las PAR y las PRE. 


\section{AGRADECIMIENTOS}

Este estudio ha sido parcialmente financiado por el Proyecto DPI2006-05531 "HPM Project-Spain: Proyecto para la Manufactura de Alto Rendimiento (High Performance Manufacturing)" del Ministerio de Educación y Ciencia. Dirección General de Investigación y por el Proyecto P08SEJ-03841 "HPM-Manufactura de Alto Rendimiento. Cuarta Ronda Internacional” de la Junta de Andalucía.

\section{BIBLIOGRAFÍA}

- Abdallah AB, Matsui Y. "JIT and TPM: Their Relationship and Impact on JIT and Competitive Performances". Actas del 9th International Asian-Pacific Decision Sciences Conference [en línea]. 2007 [consulta: 4 mayo 2009], p.1-16. Disponible en World Wide Web: <http://as.nida.ac.th/resource/asconf_ resource/apdsi2007/papers/Final_129.pdf>

- Bozarth CC, Warsing DP, Flynn BB et al. "The impact of supply chain complexity on manufacturing plant performance". Journal of Operations Management. 2009. Vol.27 p.78-93

- Cronbach L. J. "Coefficient Alpha and the internal structure of tests". Psychometrika. 1951. Vol. 16, p.297-334

- Cua KO, McKnoe KE, Schroeder RG. "Relations between implementation of TQM, JIT and TPM and manufacturing performance". Journal of Operations Management. 2001. Vol.19 p.675- 694

- Dennis P. Lean Production Simplified: A Plain-Language Guide to the World's Most Powerful Production System. 1a Edición. New York: Productivity Press, 2002. 224p. ISBN: 9781563272622

- Espolita L. "¿Qué mantenimiento debemos hacer?" DYNA Ingenieria e Industria. 2000. Vol.75 p.49-54

- Flynn BB, Schroeder RG, Flynn E.J. "World Class

Manufacturing. An Investigation of Hayes and Wheelwright's Foundation". Journal of Operations Management. 1999. Vol.17 p. $249-269$

- Fornell C, Larcker D.F. "Evaluating Structural Equation Models with Unobservable Variables and Measurement Error." Journal of Marketing Research. 1981. Vol. 18, p. 39-50.

- Forza C, Salvador F. "Information flows for high-performance manufacturing". International Journal Production Economics. 2001. Vol.70 p. $21-36$

- Galar-Pascual D, Berges-Muro L, Royo-Sanchez J. "La Problemática de la Medición del Rendimiento en la Función Mantenimiento." DYNA Ingeniería e Industria. Junio 2010. Vol. 85-5 p.429-438

- Hackman JR, Wageman R. "Total Quality Management: empirical, conceptual, and practical issues". Administrative Science Quarterly. 1995. Vol.40-2 p. 309-342

- Hallgren M, Olhager J. "Lean and agile manufacturing: external and internal drivers and performance outcomes". International Journal of Operations \& Production Management. 2009. Vol.29-10 p. 976-999.

- Huang X, Kristal MM, Schroeder R.G. "Linking learning and effective process implementation to mass customization capability". Journal of Operations Management. 2008. Vol.26 p. 714-729
- Ishikawa Karoru. What is Total Quality Control? The Japanese Way. 1a Edición. Englewood Cliffs, NJ: Prentice-Hall, 1985. 240p. ISBN: 978-0139524332

- Juran Joseph Moses, Gryna Frank. M Juran's Quality Control Handbook. 4a Edición. New York: McGraw-Hill, 1988. 1774p. ISBN: 978-0070331761

- Nakajima, Seiichi. Introduction to TPM: total productive maintenance. $1^{\text {a }}$ edicion. Cambridge: Productivity Press, 1988. 129p. ISBN: 978-0915299232

- Ohno Taiichi. Toyota Production System: Beyond Large-Scale Production. 1 ${ }^{\text {a }}$ edición. Portland: Productivity Press, 1988. 152p. ISBN: 978-0915299140

- Ortega-Jiménez CH. "Interrelación Estrategia de OperacionesTecnología y su Impacto sobre el Rendimiento de Producción: Análisis Empírico Sectorial en el Proyecto HPM Internacional". Directores: Domínguez-Machuca JA, Garrido-Vega P. Universidad de Sevilla, Facultad de Ciencias Económicas y Empresariales, 2007

- Ortega CH, Machuca JAD, Garrido P, et al. "Manufacturing Strategy and Technology Interaction: Fit Line \&t Impact". Actas del 20th Annual Conference of Production and Operation Management Society, [en línea]. 2009 [consulta: 6 mayo 2009], p. 795-849. Disponible en World Wide Web: <http://www.pomsmeetings.org/ConfProceedings/011/ FullPapers/011-0795.pdf>

- Powell TC. "Total Quality Management as competitive advantage: a review and empirical study". Strategic Management Journal. 1995. Vol.16-1 p. 15-27

- Rodriguez-Darnes P. "Participación de Mantenimiento en la Competitividad Industrial" DYNA Ingeniería e Industria. Febrero 2000. Vol. 75-1 p.10-16

- Sanchis R, Poler R, Mula J, et al. "Análisis del impacto de la Gestión de la Calidad Total en la Fabricación de Alto Rendimiento." En: Actas del 3rd International Conference on Industrial Engineering and Industrial Management - XIII Congreso de Ingeniería de Organización, (Barcelona-Terrassa, 2-4 Septiembre 2009), v.l. 2009. p. 1762- 1770. ISBN: 97884-7653-388-8

- Sanchis R, Poler R, Mula J, et al. "Análisis del Impacto del Mantenimiento Productivo Total en la Fabricación de Alto Rendimiento." En: Actas del 4th International Conference on Industrial Engineering and Industrial Management - XIV Congreso de Ingeniería de Organización, (Donostia-San Sebastián, 8-10 Septiembre 2010), v.l. 2010. p. 1177- 1186. ISBN: 978-84-95809-79-7

- Schroeder Roger, Flynn Barbara. High Performance Manufacturing-Global Perspectives. 1a edicion. New York: Wiley, 2001. 320p. ISBN: 978-0471388142 